\title{
Modalités et stratégies rhétoriques de l'éloge dans le discours publicitaire
}

\section{Marc Bonhomme}

\section{(2) OpenEdition}

1 Journals

Édition électronique

URL : http://journals.openedition.org/rhetorique/681

DOI : 10.4000/rhetorique.681

ISSN : 2270-6909

Éditeur

UGA Éditions/Université Grenoble Alpes

Édition imprimée

ISBN : 978-2-37747-062-4

\section{Référence électronique}

Marc Bonhomme, "Modalités et stratégies rhétoriques de l'éloge dans le discours publicitaire »,

Exercices de rhétorique [En ligne], 11 | 2018, mis en ligne le 08 octobre 2018, consulté le 12 septembre 2020. URL : http://journals.openedition.org/rhetorique/681 ; DOI : https://doi.org/10.4000/rhetorique. 681

Ce document a été généré automatiquement le 12 septembre 2020.

\section{(c) () (ㅇ)}

Les contenus de la revue Exercices de rhétorique sont mis à disposition selon les termes de la Licence Creative Commons Attribution - Pas d'Utilisation Commerciale - Partage dans les Mêmes Conditions 4.0 International. 


\title{
Modalités et stratégies rhétoriques de l'éloge dans le discours publicitaire
}

\author{
Marc Bonhomme
}

1 L'éloge est inhérent à l'exercice de la publicité, comme l'ont relevé de nombreux observateurs ${ }^{1}$. Mais l'éloge publicitaire présente plusieurs singularités si on le compare à d'autres pratiques laudatives. En particulier, il porte non pas sur des personnes, mais sur des produits qu'il s'agit de promouvoir par rapport à d'autres produits concurrents ${ }^{2}$. De plus, il est autocentré, dans la mesure où l'annonceur fait l'apologie de ses propres produits et plus largement de la marque dont il est le porte-parole ${ }^{3}$. Par ailleurs, l'éloge publicitaire s'inscrit dans une stratégie globale de persuasion, puisqu'il vise à susciter non seulement l'intérêt du public pour les produits promus, mais également une démarche d'acquisition et de consommation.

Cependant, par-delà ces traits élémentaires, l'éloge publicitaire constitue une réalité complexe, en ce qu'il est tiraillé entre deux tendances. D'un côté en effet, il doit s'exhiber ostensiblement dans le discours pour orienter à la hausse le message qu'il transmet et pour qu'on remarque ses effets mélioratifs sur les produits. Mais en même temps il est contraint d'estomper sa rhétorique de positivation sous peine d'apparaître comme artificiel, voire manipulateur. C'est ce double processus de monstration et de voilement de l'éloge publicitaire que nous nous proposons de mettre en évidence, en dégageant ses principales modalités à travers trois parties complémentaires. En premier lieu, nous verrons rapidement comment l'éloge publicitaire s'intègre pleinement dans le cadre rhétorique du registre épidictique. Sur cette base, nous examinerons ensuite son fonctionnement positivant dans un corpus typique représenté par un prospectus touristique. Enfin, nous approfondirons son statut paradoxal, en montrant que s'il est nécessaire à la communication publicitaire, il forme une pratique à risque, demandant des stratégies d'ajustement pour se faire accepter par le public. 


\section{1. Éloge publicitaire et registre épidictique}

3 L'éloge publicitaire existe avant tout par le modelage rhétorique de son discours. En cela, il exploite les procédés du registre épidictique ${ }^{4}$, limité à sa polarité positive ${ }^{5}$, tels qu'ils sont illustrés par une annonce promouvant le jambon de Parme :

À Parme, tout concourt à une parfaite maturation, même l'air.

L'air de Parme est idéal pour obtenir la couleur, la senteur, le goût rare et délicat des jambons de Parme, mûris quatorze mois. Il en va du jambon comme des fruits qui développent leurs meilleures qualités lorsqu'ils parviennent à complète maturation sur le lieu même de leur production. Ainsi, depuis la sélection locale très rigoureuse des viandes jusqu'à l'obtention du label de Parme garantissant l'authenticité de chacun de ses jambons, les pratiques séculaires sont respectées. Ces attentions délicates vous permettent d'accéder à une incomparable finesse et d'être subjugués par le fondant de nos jambons. À déguster sur le champ et à faire partager à vos amis.

Notre exception confirme la règle.

Label de Parme. La garantie d'une appellation d'origine contrôlée

[Campagne collective réalisée par le Ministère Italien

du Commerce Extérieur ; parution dans VSD du 14/04/2015]

\subsection{Une énonciation appréciative}

4 Le registre épidictique met en jeu une énonciation appréciative qui se concrétise par des verbes comme louer, glorifier ou exalter. Ces verbes sont généralement sousentendus dans les annonces publicitaires, à l'image de celle pour le jambon de Parme. L'énonciation appréciative du registre épidictique se distribue sur trois pôles.

5 Elle requiert un énonciateur-évaluateur qui se trouve en position haute, en raison du pouvoir discursif que lui confère l'acte de louer. Cet énonciateur-évaluateur doit en outre avoir un certain crédit pour que son entreprise d'appréciation réussisse auprès du public. Ainsi, l'annonce pour le jambon de Parme émane d'un énonciateur collectif dont la crédibilité est assurée par son statut officiel (« Ministère Italien du Commerce Extérieur »).

6 L'énonciation épidictique implique également une notion évaluée qui doit s'avérer, sous quelque aspect, digne d'éloge. Cette exigence est mentionnée par la plupart de ses analystes, dont Marc Dominicy ${ }^{6}$. En ce qui concerne le jambon de Parme, le fait qu'il soit une "appellation d'origine contrôlée " garantit, par le prestige de ce label et par son ancrage sur un terroir reconnu, ses qualités objectives.

7 L'énonciation épidictique donne aussi lieu à un discours évaluatif qui a deux caractéristiques. D'une part, il convertit la notion évaluée en objet de valeur moyennant deux opérations : la sélection d'attributs typiques et la valorisation de ces derniers de façon à les présenter comme exemplaires. Dans l'annonce considérée, le jambon de Parme est principalement décrit à travers les lieux rhétoriques de la qualité («parfaite maturation», «le goût rare et délicat») et du temps («mûris quatorze mois ", «les pratiques séculaires sont respectées ») qui non seulement le rehaussent axiologiquement, mais qui le situent au-delà des produits de même catégorie (« Notre exception confirme la règle »). D'autre part, en vue d'augmenter la portée de l'acte d'appréciation, le discours épidictique offre un important travail stylistique à la hauteur de la notion évaluée. Ce travail repose sur des techniques d'amplification qui sont destinées à "magnifier [...] l'objet du discours ${ }^{8}$ » et qui se traduisent par des 
modalités expressives variées dans l'annonce pour le jambon de Parme: forte "qualification attributive" " par la réitération d'adjectifs évaluatifs ("idéal», «délicates »), de superlatifs (" leurs meilleures qualités ", "très rigoureuse ») et de substantifs abstraits (« authenticité », finesse ») ; recours aux figures de la comparaison («Il en va du jambon comme des fruits [...]) et de l'hyperbole (« une incomparable finesse ») ; gradation syntaxique ternaire (« la couleur, la senteur, le goût rare et délicat des jambons de Parme »)...

\subsection{Une stratégie de séduction}

Ce discours appréciatif est ordinairement suivi d'une réaction rapide de la part de ses destinataires, comme l'indique la fin de l'annonce pour le jambon de Parme: "À déguster sur le champ ». Cette réception rapide est favorisée par le déficit interactif de la communication épidictique, vu qu'à travers elle "on donne juste à connaître les bonnes [...] qualités du sujet que l'on traite ${ }^{10} »$. Chaïm Perelman et Lucie OlbrechtsTyteca précisent que celle-ci s'adresse en effet à des récepteurs qui n'ont pas à prendre position sur le spectacle de paroles auquel ils assistent ${ }^{11}$. Un tel déficit interactif se double d'un déficit argumentatif. Comme le souligne Christian Plantin ${ }^{12}$, alors que l'argumentation suppose une question à discuter et des décisions à prendre, la communication épidictique est dans la majorité des cas dépourvue de débats consécutifs. Toutefois, si elle se dérobe à l'argumentation rationnelle classique, elle n'en est pas moins persuasive, mettant en œuvre une stratégie de séduction dans l'acception de Jean-Blaise Grize ${ }^{13}$. D'ordre infraréflexif, cette dernière recourt au maniement des valeurs et à leur logique impressive, fondée sur des éclairages incitatifs.

9 C'est précisément ce qui se passe avec le registre épidictique. En transformant la notion évaluée en objet de valeur, l'énonciateur fait tout pour que le destinataire apprécie celui-ci dans le même sens que lui. Si elle réussit, cette appréciation partagée se traduit par une séduction cognitive du destinataire, susceptible de déclencher son agrément au discours tenu. Ainsi, dans l'annonce pour le jambon de Parme, on est « subjugué » avant de «déguster ». On peut alors parler d'une communication empathique qui passe directement du dire de l'énonciateur au croire de l'énonciataire. Cette communication est en même temps esthétique, en ce que le discours évaluatif, lui-même valorisé stylistiquement comme on l'a vu, a également de fortes chances d'exercer une séduction formelle sur ses destinataires ${ }^{14}$.

\section{2. Étude de cas : la mise en scène de l'éloge dans une plaquette touristique pour la ville de Berne}

10 L'examen détaillé d'une plaquette touristique diffusée en 2010 par Bern Tourismus et promouvant la ville de Berne, capitale de la Suisse, permet de voir plus précisément comment les modalités et les stratégies épidictiques en élaborent une figuration idéalisée. Transformant la réalité de la ville en une représentation plus ou moins imaginaire de façon à en accroître l'attractivité, cette plaquette de douze pages, organisée en cinq planches intérieures de format A3, met en scène une rhétorique de l'éloge qui s'appuie sur trois procédures: une stratégie préliminaire de schématisation ${ }^{15}$ et une stratégie principale de positivation, le tout prenant place dans une visée d'adhésion du public. 


\subsection{Schématisation de la ville}

11 Schématiser la ville, c'est en retenir les aspects les plus caractéristiques à travers deux modalités énonciatives.

\section{Sélectionner}

Foncièrement elliptique, la plaquette considérée se limite au cinquième de la ville de Berne, à savoir son centre historique circonscrit par la boucle de l'Aar. Ce processus synecdochique, qui fait de la partie centrale un condensé du tout, se rencontre aussi bien dans le texte que dans les grandes vignettes illustratives. Ces dernières présentent tantôt une configuration centripète, le reste de la ville (comme la colline du Gurten, planche 4) irradiant vers le centre dans une perspective axiale, tantôt une composition centrifuge, le centre se diffusant vers la périphérie de la ville et son environnement. Ainsi, sur la planche 5, la cathédrale au premier plan fournit le pivot iconique de l'illustration qui se prolonge à l'arrière-plan par les montagnes de l'oberland bernois.

\section{Emblématiser}

Par cette modalité énonciative, le segment central de la ville de Berne ainsi sélectionné met en exergue des scènes ou des lieux représentatifs à forte prégnance mémorielle. Si l'on prend la première planche de la plaquette, la vieille ville est fractionnée en trois sous-ensembles emblématiques. On observe d'abord une grande vignette qui occupe les deux tiers de la planche et qui exemplarise, en une sorte de carte postale intemporelle, le côté est du centre-ville. On constate ensuite sur la partie supérieure de la planche une séquence horizontale de neuf vignettes moyennes qui narrativisent visuellement certains aspects saillants du centre-ville (marché sur la Bärenplatz, commerçant devant son échoppe...). Enfin, on distingue une séquence verbo-iconique verticale qui achève d'encadrer la grande vignette sur le côté droit de la planche et dont les trois microvignettes illustrent les pavés textuels qui leur sont accolés par des représentations urbaines remarquables. Entre autres, l'image de la coupole du parlement ancre thématiquement et balise visuellement le second pavé textuel consacré aux activités politiques de la capitale qu'est Berne.

14 Cette disposition à la fois tabulaire et séquentielle de la première planche de la plaquette fait voir autant de figures significatives dont chacune symbolise Berne. Parmi elles, on relève le blason de la ville (deuxième vignette de la séquence horizontale supérieure), l'un ou l'autre de ses monuments historiques, comme la fontaine de l'Ogre (troisième vignette de la séquence verticale), ou son animal fétiche : l'ours. Celui-ci est montré en gros plan (sixième vignette horizontale), puis décrit dans le texte qui fonctionne alors comme relais ${ }^{16}:$ "La ville avec sa fosse aux ours tant visitée - l'ours étant l'animal héraldique de la ville [...] ».

\subsection{Positivation de la ville}

15 Si par la maitrise de ses filtrages, la schématisation de la plaquette contribue déjà à la célébration de Berne, celle-ci trouve son aboutissement dans l'inventio (création de 
valeurs) et dans l'elocutio (techniques rédactionnelles) des auteurs de cette plaquette, à travers une stratégie de positivation qui met en œuvre deux modalités énonciatives.

\section{Valoriser} convertissent en un espace axiologisé, en lui attribuant des valeurs d'usage et des valeurs symboliques. Dans la plaquette de Bern Tourismus, les valeurs d'usage - qui définissent l'évaluation pratique du produit-ville - sont secondaires. Celles-ci concernent par exemple la grande accessibilité de Berne, exposée dans le premier pavé textuel de la planche 5 :

Des liaisons directes avec les trains à grande vitesse TGV, ICE et Pendolino existent depuis les pays voisins. De plus, Berne se situe près des aéroports intercontinentaux de Zurich, Genève et Bâle. Et l'aéroport international de Berne-Belp se trouve quasiment devant la porte de la ville. Avec des liaisons quotidiennes vers de nombreuses villes européennes d'importance.

Par contre, la ville de Berne est investie d'importantes valeurs symboliques, formulées au moyen de couplages antithétiques :

- Singularité/Universalité. S'intégrant dans le lieu rhétorique de l'« unique ${ }^{17}$ », la singularité met en valeur quelques curiosités propres à Berne: "Une attraction sans pareille est la foire aux Oignons » (pl. 2) - «Les pièces rares et exclusives de son musée des Beaux-Arts» (pl.4)... Quant à l'universalité, elle crée une valorisation ouvrant Berne sur les flux internationaux. C'est le cas avec la mention de l'inscription de la ville au " patrimoine mondial de l'UNESCO ", ou avec les allusions à ses célébrités que sont Klee (« la plus grande collection de travaux de Paul Klee », pl. 4) et Einstein : « De 1902 à 1909, une autre personnalité a aussi vécu à Berne: Albert Einstein. Il y a élaboré sa théorie de la relativité restreinte » $(\mathrm{pl} .4)$.

- Passé/Présent. Selon les rédacteurs de la plaquette, Berne condense également les valeurs temporelles de la tradition et du modernisme. Ces valeurs antithétiques sont clairement proclamées sur la planche 4 consacrée à la diversité du tissu urbain bernois : «On veille à conserver le paysage urbain de la vieille ville. [...] Berne est aussi ouverte aux tendances modernes de l'architecture et du design. " Ces valeurs apparaissent encore dans le récapitulatif de la dernière planche: «Les boutiques de la vieille ville abondent en antiquaires qui satisferont les plus exigeants. L'Université construit actuellement des instruments pour la sonde Rosetta en vue de son voyage vers la comète Wirtanen.

- Culture/Nature. La ville de Berne est enfin décrite comme concentrant les valeurs davantage thématisées d'une riche vie culturelle: musées («Berne vous propose 16 musées déclinant des thèmes allant de l'histoire à la communication », pl. 4), activités musicales et théâtrales: "Le théâtre municipal et le Kulturcasino se trouvent à quelques pas l'un de l'autre» (pl.4)... Cette vie culturelle cohabite avec une nature restée intacte, comme en témoignent les nombreuses informations sur les espaces verts. Ces derniers sont largement représentés sur les illustrations (comme les grandes vignettes des planches 3 et 4) et abondamment commentés dans les différents pavés textuels : «Le paysage créé par la nature est idéal pour les activités sportives de toutes sortes » (pl. 3)...

La ville de Berne apparaît ainsi comme un espace de totalisation, fortement positivé et riche en polarités axiologiques. Celles-ci ne s'y côtoient pas seulement, mais elles y 
fusionnent en de nombreux échanges. Berne se transforme alors en un espace de médiation assurant un syncrétisme entre les valeurs les plus diverses, ce que laissent entendre plusieurs titres de la plaquette: «Un patrimoine ancestral resté jeune » (pl. 1), « Citoyens du monde heureux au détour de petites ruelles » (pl. 4)...

\section{Esthétiser} traits stéréotypés : lieu microcosmique idéalisé, esthétisé et euphorique. Mais tandis que le locus amœnus classique se caractérise par sa fermeture (pensons à la plaine du Forez dans L'Astrée d'Honoré d'Urfé), le locus amœnus urbain construit par les concepteurs de cette plaquette est ouvert sur l'extérieur, comme l'indique l'un des titres de la planche $5:$ « Berne : la capitale au cœur de la Suisse, au cœur de l'Europe ».

\subsection{De l'épidictique au délibératif}

22 À travers sa scénographie laudative, une telle plaquette touristique a un but fondamental : attirer les visiteurs à Berne. Comme on l'a précisé, le discours épidictique comporte une orientation persuasive, prédisposant ses destinataires à l'action. Mais en plus dans les textes publicitaires, il est accompagné par des marques du registre délibératif, basées sur le conseil ${ }^{19}$.

\section{Faire adhérer}

L'objectif de cette plaquette est de susciter l'adhésion des lecteurs aux valeurs idéalisées et séduisantes de Berne, de sorte qu'ils aient envie de venir les découvrir sur 
place ${ }^{20}$. Pour stimuler cette adhésion, la plaquette s'appuie sur une stratégie centrale qui consiste à favoriser l'identification des lecteurs avec les flâneurs qu'elle met en scène à plusieurs reprises.

$\mathrm{Au}$ niveau des pavés rédactionnels, divers pronoms indéfinis précèdent les verbes introduisant la description de la ville : «À quelques minutes de la gare, on est au bord de l'Aar, entouré de nature. En suivant la rivière, on longe bientôt le jardin botanique. En le remontant, on arrive au zoo Dählhölzli» (pl.3)... De même, cette description s'effectue fréquemment par le biais d'une focalisation zéro, qu'elle recoure à des formulations impersonnelles ("Un réseau d'arcades où il fait bon se promener », pl. 2) ou à l'effacement du pôle agent : «La marche à pied sur les promenades de l'Aar est appréciée » (pl.3). De tels procédés instaurent une référence ouverte dans laquelle n'importe qui peut se reconnaître : la population bernoise, mais aussi les touristes et les visiteurs éventuels, dont les lecteurs de la plaquette. Parallèlement, certaines illustrations, comme la grande vignette de la planche 2, font voir des promeneurs de dos, que ce soit sous les arcades ou sur la place du Marché. Cette représentation de promeneurs anonymes engendre un effet de prolepse, à travers lequel ces derniers pourraient bien être déjà les lecteurs eux-mêmes en train de consommer la ville.

\section{Conseiller}

L'incitation faite aux lecteurs à participer au locus amœnus urbain célébré donne également lieu à des formulations plus explicites qui appartiennent au registre délibératif du conseil. Au niveau des pavés rédactionnels, le conseil est encore diffus, prenant la forme de modalités sollicitatives, avec une présence claire du pôle allocutif vous : «Les fameuses arcades vous engagent à la promenade » (pl. 1) - « La propreté des flots vous incite à la baignade ${ }^{21} »(\mathrm{pl} .3) .$. Mais dans la rubrique « Notre tuyau » de la planche 2 , le conseil devient plus pressant avec des modalités directives à l'impératif : «Prélassez-vous lors d'un lèche-vitrine le long des centaines de boutiques». De surcroît, sur la page de dos de la couverture, le conseil concerne plus particulièrement le contact avec l'office de Tourisme bernois, dans une amorce d'interaction dialogique : « Nous sommes là pour vous pendant les 365 jours de l'année. Demandez-nous d'autres tuyaux et idées».

\section{L'éloge publicitaire comme pratique à risque}

Si l'éloge paraît aller de soi dans la communication publicitaire, il s'agit en fait d'une pratique risquée, toujours susceptible d'échecs lors de sa réception. Il convient à présent d'examiner de plus près les difficultés auxquelles est confronté le registre épidictique en publicité, ainsi que les stratégies employées par les publicitaires pour les résoudre autant que possible.

\subsection{Les problèmes de l'éloge publicitaire}

Nous avons insisté sur l'idée que l'éloge suppose une réalité méritant d'être louée et que le registre épidictique utilisé doit être approprié à son référent. Une telle appropriation est certes assurée avec la charcuterie haut de gamme comme le jambon de Parme ou une ville touristique comme Berne, mais la publicité doit aussi promouvoir 
de nombreux produits moins réputés ou très ordinaires, auquel cas leur éloge peut devenir déplacé. Ce risque est manifeste avec la réclame du XIX ${ }^{e}$ siècle, dont la rhétorique grandiloquente semble souvent peu en rapport avec la qualité effective des produits qu'elle loue. Soit cet extrait d'un prospectus rédigé par César Birotteau dans le roman du même nom de Balzac, lequel célèbre la Pâte des Sultanes et l'Eau Carminative inventées par ce parfumeur :

Double Pâte des Sultanes et Eau Carminative de César Birotteau

Découverte merveilleuse approuvée par l'Institut de France

Cette Pâte et cette Eau possèdent d'étonnantes propriétés pour agir sur la peau, sans

la rider prématurément [...].

Cette précieuse Pâte, qui exhale les plus doux parfums, fait disparaître les taches de rousseur les plus rebelles, blanchit les épidermes les plus récalcitrants, et dissipe les sueurs de la main dont se plaignent les femmes non moins que les hommes.

L'Eau Carminative enlève ces légers boutons qui, dans certains moments, surviennent inopinément aux femmes et contrarient leurs projets pour le bal [...].

La Double Pâte des Sultanes et l'Eau Carminative sont deux compositions opérantes, d'une puissance motrice agissant sans danger sur les qualités internes et les secondant; leurs odeurs essentiellement balsamiques et d'un esprit divertissant réjouissent le cœur et le cerveau admirablement, charment les idées et les réveillent ; elles sont aussi étonnantes par leur mérite que par leur simplicité.

[Honoré de Balzac, César Birotteau, 1837]

Ce prospectus constitue un bel exemple de texte publicitaire épidictique, à l'écriture très littéraire. Mais le cumul des emphases qualificatives ("étonnantes propriétés ", "précieuse Pâte »), des superlatifs («les plus doux parfums») et des adverbes hyperboliques ("admirablement») lui confère une tonalité outrancière suspecte. De plus, la capacité de ces produits à résoudre tous les problèmes de soin, amplifiée en des listes mêlant des considérations scientifiques et esthétiques, est explicitement rattachée au merveilleux (" découverte merveilleuse»), dans une magie du verbe qui confine au charlatanisme. Par-delà le genre très daté de la réclame, les éloges forcés abondent dans les publicités modernes, à l'instar de cette annonce pour les détergents ménagers Potz :

Top qualité. Une technologie très moderne de flacon, un design parfait et une mise en valeur rare, telles sont les caractéristiques de la nouvelle gamme Fresh Power de Potz.

[Parution dans L'Hebdo du 08/10/2016]

On perçoit aisément dans cette annonce un discours exagéré qui maximalise discursivement une réalité banale (une certaine innovation dans le domaine du flaconnage), cette réalité détonnant avec l'intensification verbale que lui accorde l'annonceur.

Cette rhétorique épidictique exaltant excessivement les qualités des produits n'a pas manqué d'être dénoncée comme une pure fiction par divers observateurs. Ainsi, Jean Baudrillard reproche à la publicité de recourir à «la logique du père Noël» et à «la magie du cargo ${ }^{22}$ ». Selon François Brune, l'omniprésence de l'éloge dans la publicité aboutit à une déperdition de la fonction dénotative du langage: "Cette perpétuelle surenchère verbale des publicités finit par faire perdre la juste mesure des choses. La raison s'égare dans des signes exubérants qui ne renvoient plus qu'à eux-mêmes ${ }^{23}$ ". Pour sa part, Edgar Morin en vient à comparer les pratiques idéalisantes de la publicité à une drogue : "La publicité transforme le produit en stupéfiant mineur - ou elle lui inocule la substance droguante, de façon que son achat-consommation procure immédiatement l'euphorie-soulagement et à long terme l'asservissement ${ }^{24}$ ». 
30 Le problème est plus délicat lorsque la nécessité de l'éloge conduit certains publicitaires à dissimuler les dangers présentés par leurs produits, en diffusant des " affirmations partisanes qui [les] habillent de vertus excessives ${ }^{25}$ ». L'éloge devient alors clairement manipulateur, comme le montre l'annonce suivante pour la compagnie EDF :

Centrale nucléaire de Saint-Laurent-des-Eaux. Production quotidienne de $\mathrm{CO} 2$ : zéro. Production annuelle de $\mathrm{kWh}$ : douze milliards.

Chez EDF, le soin de l'environnement est capital. Ainsi, en France plus de $90 \%$ de l'électricité est produite sans émettre de $\mathrm{CO} 2$, gaz responsable de l'effet de serre. Et ceci, essentiellement grâce à l'énergie nucléaire. En vingt ans, la pollution a été réduite de $30 \%$ et la France est devenue le pays où l'atmosphère est la moins polluée par la production d'énergie.

[Parution dans Le Point du 05/03/2004]

31 Cette annonce fait l'apologie de l'électricité nucléaire en convertissant un aspect positif très périphérique de celle-ci (non émission de $\mathrm{CO} 2$ ) en argument principal. Mais cette apologie repose sur une ellipse généralisée - et difficilement acceptable - de tous les aspects négatifs d'une telle énergie : risque de pollution atomique, dangers sanitaires, problèmes de stockage des déchets radioactifs... On a affaire à une publicité de contreconnotation dont la stratégie consiste à inverser la doxa hostile au nucléaire et à jouer sur un contrat de véridiction flou. Elle est explicitement non mensongère, vu que les centrales nucléaires produisent effectivement peu de $\mathrm{CO} 2$. En même temps, elle est implicitement mensongère, puisqu'il est scientifiquement prouvé que l'électricité nucléaire est une menace pour l'environnement. Cette publicité s'inscrit dans la mouvance de l'éco-blanchiment, largement attestée depuis quelques décennies et dénoncée par de nombreuses associations, qui consiste à exploiter tendancieusement la valorisation écologique ${ }^{26}$.

L'éloge publicitaire est confronté à un autre risque : celui de ne pas être interprété comme tel par ses destinataires. Cela arrive lorsque l'annonceur contrôle mal les connotations valorisantes développées dans sa publicité et qu'elles sont interprétées comme dévalorisantes. Dans son ouvrage Quand la publicité est aussi un roman, Pierre Lemonnier rappelle l'accueil très mitigé reçu il y a quelques années par le slogan pour l'eau minérale Évian : «L'eau neuve de vos cellules ${ }^{27}$ ». Censé insuffler une connotation de régénérescence au produit, ce slogan a au contraire évoqué les cellules cancéreuses - et donc la maladie - dans la mémoire du public, ce qui a annihilé ses effets épidictiques. Soit encore cette publicité pour l'Ice Tea de la firme suisse Migros :

Teatanic.

Ice Tea Peach et Ice Tea Lemon. Migros.

[Parution dans TV8 du 05/12/1997]

Diffusée en 1997 juste après la sortie du film Titanic de James Cameron, cette publicité centrée sur le mot-valise métaphorique Teatanic (tea + Titanic) construit son éloge du produit en récupérant les valeurs de célébrité et de mode transmises par le film. Mais cette production métaphorique a suscité chez certains lecteurs des inférences axiologiques négatives dues aux thèmes dramatiques de ce film (naufrage, mort...), ce qui a neutralisé du même coup ses effets valorisants. 


\subsection{Favoriser une bonne réception de l'éloge}

\section{décalage sur l'axe de la communication, il devient le fait du consommateur du produit,} n renforcer la légitimité à l'exemple d'une publicité pour l'agence de voyage Fram :

Les grands circuits, avec Fram, moi j'aime... Cet été j'ai fait un circuit la Réunion-île Maurice... Et il y a un mois ... La Chine ! Bien sûr, on m'avait dit que l'on voyait la grande muraille de la lune! Alors la contempler, vous imaginez... Pékin et la Cité interdite... 2 nuits à Shangaï dont je rêvais depuis toujours... Xian la capitale historique... Mais mon plus beau souvenir c'est une petite croisière sur la rivière Li, un paysage renversant, comme une estampe chinoise... Et puis le dernier soir, un dîner inoubliable dans un restaurant flottant à Aberdeen... Super circuit à travers toute la Chine, vous voulez vraiment un bon conseil... Offrez-vous un circuit de 15 jours en Chine, c'est chez Fram. C'est inoubliable, vous verrez !

[Parution dans L'Express du 25/04/2012]

Outre la garantie d'expérience qu'un tel témoignage cherche à fournir, il présente l'avantage de valoriser le produit par son efficacité éprouvée, dans une anticipation de la satisfaction du client. Une autre stratégie consiste à louer le produit en faisant appel à la caution d'une vedette. Selon cette perspective, les assurances Nationale Suisse sollicitent les services du champion de tennis Roger Federer :

Roger Federer : un parfait ambassadeur pour Nationale Suisse.

Nous avons pu nous adjoindre un véritable artiste pour notre campagne: Roger Federer, meilleur joueur mondial et sportif mondial de l'année à plusieurs reprises, suscite autant d'enthousiasme par ses prestations sportives que par son attitude sympathique et modeste hors des courts de tennis. Ces qualités en font un parfait ambassadeur pour Nationale Suisse puisqu'il incarne mieux que quiconque nos valeurs phares : individuel, efficace, sympathique.

[Parution ans L'Hebdo du 20/06/2017]

Le recours à Roger Federer offre un argument d'autorité qui valide la présentation élogieuse de cette compagnie d'assurances. L'éloge est ici d'autant plus motivé qu'il établit une analogie entre les qualités reconnues de ce champion et le produit promu.

Sur le plan de leur composition, les annonces publicitaires mettent en œuvre des stratégies textuelles pour donner davantage de vraisemblance aux éloges qu'elles renferment, surtout lorsqu'ils prennent la forme d'hyperboles. Ainsi, l'impression d'exagération engendrée par certains slogans se voit rectifiée et nuancée par le pavé rédactionnel consécutif, à l'image de l'annonce ci-après : 
Achetez le soleil et la mer.

Avec un étage pour les Balandrins, deux étages pour les Maisons de Pêcheur, de trois à cinq pièces, chaque maison de Port-Grimaud s'ouvre sur le soleil et la mer.

[Parution dans VSD du 15/05/1996]

Le slogan "Achetez le soleil et la mer » constitue une amplification hyperbolique des résidences secondaires vantées par cette annonce, moyennant une métonymie spatiale Environnement/Produit. Pertinente dans un slogan dont la fonction est d'exagérer, cette amplification élogieuse est ensuite réajustée à l'aide d'une reformulation littérale: "chaque maison [...] s'ouvre sur le soleil et la mer». On a de la sorte une stratégie de rattrapage qui naturalise dans une séquence descriptive un premier énoncé emphatique destiné à attirer l'attention du lecteur. De même, les éloges publicitaires gagnent en vraisemblance lorsqu'ils sont accompagnés de développements explicatifs, comme dans cette annonce :

Mitsubishi Pajero. Monstrueusement suréquipé.

Équipements offerts Pajero XStorm : Sellerie cuir noir, alcantara gris clair - Barres de toit type aluminium - Intérieur type aluminium avec compteurs blancs - Calandre type sport - Projecteurs fumés.

Équipements de série Pajero XStorm: Motorisation 3.2160 ch Turbo Diesel - Système de navigation à technologie DVD (couverture Europe) - Airbags frontaux et latéraux - Climatisation automatique - Boîte automatique et séquentielle - Système actif de contrôle de trajectoire MASC et antipatinage MATC - Contrôle assisté du frein moteur EBAC - Toit ouvrant électrique - Sièges avant chauffants - ABS avec EBD.

[Parution dans Le Nouvel Observateur du 21/04/2005]

Cette annonce débute par un slogan qui proclame la nature exceptionnelle du produit grâce à deux hyperboles: "Monstrueusement suréquipé ». Mais la longue liste des équipements qui lui fait suite permet de justifier, par son étendue et sa précision, le bien-fondé de cette hyperbolisation.

Sur le plan référentiel enfin, de nombreuses publicités délaissent les éloges idéalisants, considérés comme peu adaptés, pour étayer leurs éloges par la « vérité » des produits. Cette stratégie relativise la rhétorique épidictique, mais elle la rend aussi plus authentique, en valorisant les produits par leurs caractéristiques économiques intrinsèques, promues en arguments de vente. Une telle démarche s'observe dans les publicités pour les grandes surfaces :

La meilleure des promotions commence par une information compréhensible par tous. Sur un emballage Leclerc Marque Repère, vous apprendrez qu'un verre de pur jus d'orange participe à couvrir les besoins journaliers d'un enfant en vitamine $\mathrm{C}$ et que 40 gr. de camembert contiennent la même quantité de calcium qu'un yaourt nature.

[Parution dans VSD du 11/03/2014]

D'autres annonces n'hésitent pas à recourir à la face négative du registre épidictique qu'est le blâme, en principe proscrit du discours publicitaire. Ainsi, les magasins Casino font état des critiques des consommateurs à l'encontre de leurs produits :

Enquête SOFRES

$31 \%$ d'entre vous n'ont pas aimé nos raviolis.

Voici les nouveaux raviolis Casino.

Ils sont bien plus savoureux et vous y êtes pour beaucoup. Produits Casino

[Parution dans Femme Actuelle du 18/07/2010]

41 Le blâme est certes ici retourné en éloge et la communication demeure sous le contrôle de l'annonceur. Mais outre qu'elle introduit un vernis réaliste et l'impression du parler- 
vrai dans l'annonce, cette stratégie interactive avec le public suscite une valorisation implicite de la marque, puisqu'elle se montre attentive à ses réactions.

\section{Stratégies de brouillage}

Conjointement aux mises en texte étayant les pratiques publicitaires élogieuses, on relève au contraire une tendance récente à les estomper par une systématisation du second degré, typique des publicités dites " postmodernes ${ }^{28}$ ». L'éloge asserté comme tel laisse alors la place à des configurations épidictiques floues dans leurs formes et ambiguës dans leur interprétation. Celles-ci répondent à des stratégies de brouillage qui se traduisent principalement par deux réalisations.

L'éloge publicitaire emprunte de plus en plus les décalages énonciatifs de l'humour ${ }^{29}$, à travers lesquels les annonceurs prennent du recul avec la rhétorique épidictique conventionnelle. Au degré faible, l'éloge - pratique sérieuse, car indispensable à la promotion des produits - est traité selon une modalité non sérieuse qui le convertit en un exercice ludique, apparemment détaché de tout souci de persuasion. C'est le cas lorsque l'annonceur multiplie les calembours dans sa présentation du produit :

Madrid $210 €$. Ce prix est valable pour l'olé et retour.

Marrakech $260 €$. Ça vous en babouche un coin.

New York $430 €$. Les gratte-ciel à prix gratte sol.

Circuit Big Ouest, 20 jours aux USA $640 €$. Circuit typique à prix tout pitit ${ }^{30}$.

1 villa en Floride $525 €$. Profitez des bonnes occases de l'oncle Tom.

GO Voyages

[Parution dans VSD du 15/06/2001]

Ou quand l'annonce semble se limiter à un jeu sur des variations néologiques :

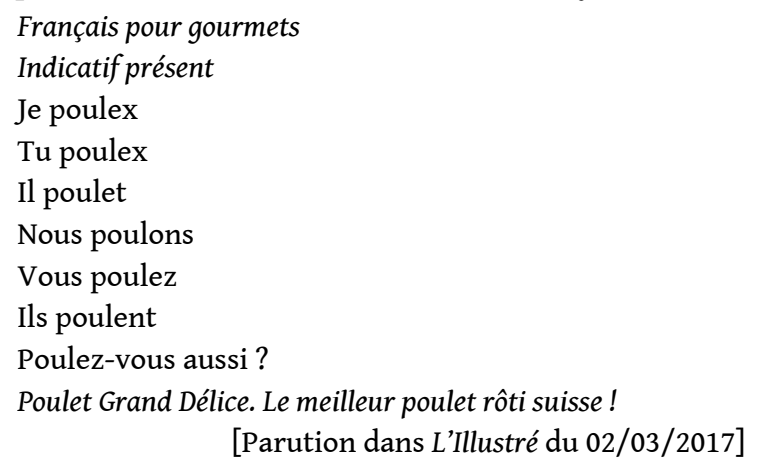

Dans ces exemples, on assiste à une distanciation par rapport à l'écriture épidictique canonique, en ce que le travail sur les mots y voile la fonction informative du langage. Cependant, l'éloge des produits est bien présent, quoique d'une façon décalée, comme le montre l'annonce GO Voyages où la déclinaison des calembours ne manque pas d'évoquer l'intérêt des destinations proposées. De plus, par leur éloge indirect des produits, ces annonces jouent sur l'importance du style dans le registre épidictique, recherchant de surcroît l'approbation élogieuse du public à propos de la performance humoristique des publicitaires. Au degré fort, l'humour introduit une distanciation ludique avec le processus épidictique lui-même. Alors que celui-ci demande une convenance assumée entre la tonalité discursive adoptée et la notion louée, cette convenance peut subir un double dérèglement. Tantôt l'annonceur feint de mettre en doute la pertinence de l'éloge, en l'affectant d'une modalité affabulatoire et en jouant avec l'accusation de mensonge portée contre les hyperboles publicitaires : 
MYTHOMANE (mitoman) adj. et n. - 1900 ; de mytho- et suff. -mane. Qui est atteint de mythomanie - Subst. voir fabulateur, menteur. Exemple : "Il m'a dit qu'il s'était acheté une Golf avec la climatisation de série, l'ABS avec répartiteur électronique de série et 4 airbags de série pour $99900 \mathrm{~F}$, c'est un mythomane ».

Volkswagen

[Parution dans Le Point du 19/06/1999]

Tantôt l'annonceur fait semblant de transgresser les conventions épidictiques qui veulent que l'éloge soit orienté à la hausse. Les qualités du produit promu se voient dès lors décrites comme sans importance :

IBM. Nouveau NetVista X40.

Il est beau, mais c'est un détail.

[Parution dans L'Hebdo du 17/10/2010]

Ces annonces constituent des productions épidictiques composites, fondées sur la réappropriation d'hypotextes considérés comme connus du public. Grâce à cette stratégie de réappropriation, la publicité estompe son contenu commercial et acquiert une plus-value culturelle. En cela, ces pratiques parodiques valorisent l'énonciateur publicitaire qui exhibe son savoir à travers le montré-caché de leurs mises en texte. Elles créent en même temps une complicité avec les lecteurs, transformés en partenaires dans le partage d'un interdiscours attractif, propre à emporter leur adhésion. Mais ces éloges publicitaires par vampirisation parodique ne sont pas dépourvus de risques. D'une part, la notoriété de l'hypotexte détourné tombe à plat pour les lecteurs qui n'identifient pas ce dernier. D'autre part, il arrive que la célébrité de l'hypotexte (cas de l'aphorisme de Descartes) occulte l'éloge du produit, rejeté à l'arrière-plan du discours. Surtout, l'hybridation intertextuelle d'univers différents peut introduire des incohérences dans le discours épidictique. Si l'on prend l'annonce 
parodique Raynal et Roquelaure, on remarque une contradiction dans la relation causeconséquence qui la structure : "c'est la faute de », négatif et issu de l'hypotexte de V. Hugo, est en effet anti-orienté avec « on se régale », positif et spécifique à l'hypertexte publicitaire.

\section{Conclusion}

L'éloge publicitaire repose finalement sur une visée pragmatique constante : valoriser les produits présentés de façon à les rendre désirables pour un public lui-même valorisé par leur acquisition. Toutefois, cette visée intrinsèque donne lieu à des stratégies et à des modalités discursives diversifiées, suivant la nature des produits promus et l'évolution des pratiques publicitaires. D'un côté, il est plus facile de promouvoir une destination touristique que l'électricité nucléaire par des stratégies idéalisantes et par des procédés amplifiants éprouvés depuis longtemps. D'un autre côté, avec la culture publicitaire croissante du public et le développement de son esprit critique, l'éloge publicitaire est davantage contraint de justifier ses pratiques et de les diluer dans des stratégies indirectes. Mais à condition que les annonceurs maîtrisent convenablement leur discours, ces nouvelles formes restent épidictiques, car on sait que dès qu'ils prennent la parole, c'est pour célébrer les produits qui les font vivre.

Sur un autre plan, quelles que soient les stratégies et les modalités qu'elle adopte, la démarche laudative de la publicité s'avère profondément rhétorique. En effet, cette démarche perpétue à sa façon les trois grands préceptes constitutifs de la rhétorique, qui ont été formulés dans l'Antiquité latine ${ }^{33}$, tout en leur accordant un statut inégal : si elle privilégie le delectare (plaire à travers l'éloge) et le movere (emporter affectivement l'adhésion par l'éloge), le docere n'est pas négligé, pour peu qu'elle évite les pièges de la sophistique, en prenant aussi en compte la dimension informative des produits qu'elle exalte. Mais c'est toujours au lecteur qu'il revient en dernier ressort de contrôler et d'évaluer si ce pacte qui régit la communication publicitaire est bien respecté.

\section{BIBLIOGRAPHIE}

Adam J.-M. et Bonhomme M., L'Argumentation publicitaire, Paris, Armand Colin, 2014.

Aristote, Rhétorique, éd. B. Timmermans, Paris, Le Livre de Poche, nº 4607, 1991.

Barthes R., «Rhétorique de l'image », Communications, n 4, 1964, p. 40-51.

Baudrillard J., Le Système des objets, Paris, Gallimard, 1968.

Baudrillard J., La Société de consommation, Paris, Denoël, 1970.

Bonhomme M., « Parodie et publicité », Tranel, n 44, 2006, p. 165-180.

Bonhomme M., « De la pragmatique à la stylistique du registre épidictique », dans L. GaudinBordes et G. Salvan (éds), Les Registres, Louvain-la-Neuve, Bruylant-Academia, 2008, p. 79-92. 
Bonnange C. et Thomas C., Don Juan ou Pavlov, Paris, Le Seuil, 1987.

Brune F., Le Bonheur conforme, Paris, Gallimard, 1985.

Cathelat B. et Cadet A., La Publicité, de l'instrument économique à l'institution sociale, Paris, Payot, 1968.

Cicéron, L'Orateur, éd. A. Yon, Paris, Les Belles Lettres, 1964.

Dominicy M., «L'épidictique et la théorie de la décision », dans M. Dominicy et M. Frédéric (éds), La Mise en scène des valeurs, Lausanne/Paris, Delachaux et Niestlé, 2001, p. 49-77.

Everaert-Desmedt N., La Communication publicitaire, Louvain-la-Neuve, Cabay, 1984.

Gibert B., La Rhétorique ou les règles de l'éloquence, Paris, D’Espilly, 1749.

Grize J.-B., « L'argumentation : explication ou séduction », dans L'Argumentation, Lyon, Presses

Universitaires de Lyon, 1981, p. 29-40.

Grize J.-B., Logique et langage, Paris, Ophrys, 1990.

Lemonnier P., Quand la publicité est aussi un roman, Paris, Hachette, 1985.

Libaert Th, La Communication verte : l'écologie au service de l'entreprise, Paris, Éditions Liaisons, 1992.

Meyer M., Questions de rhétorique, Paris, Le Livre de Poche, n 4171, 1993.

Molinié G., Dictionnaire de rhétorique, Paris, Le Livre de Poche, n 8074, 1992.

Perelman Ch. et Olbrechts-Tyteca L., Traité de l'argumentation, Bruxelles, Éditions de l'Université Libre de Bruxelles, 1988.

Plantin C., L'Argumentation, Paris, PUF, « Que sais-je ? », 2005.

Reboul O., La Rhétorique, Paris, PUF, « Que-sais-je ? », 1984.

Riou N., Pub Fiction, Paris, Éditions d'Organisation, 1999.

Soulages J.-Cl., « Les stratégies humoristiques dans le discours publicitaire », Questions de communication, $\mathrm{n}^{\circ} 10,2006$, p. 103-118.

Spitzer L., «La publicité américaine comme art populaire », Poétique, n³4, 1978, p. 152-171.

Sullivan D., « The Ethos of Epideictic Encounter », Philosophy and Rhetoric, n² 26, 1993, p. 113-133.

Teyssier J.-P., Frapper sans heurter. Quelle éthique pour la publicité ?, Paris, Armand Colin, 2004.

Viala A., « Des “registres” ", Pratiques, n 109-110, 2001, p. 165-177.

\section{NOTES}

1. Voir notamment N. Everaert-Desmedt, La Communication publicitaire, Louvain-la-Neuve, Cabay, 1984 ; C. Bonnange et C. Thomas, Don Juan ou Pavlov, Paris, Le Seuil, 1987 ; ou J.-M. Adam et M. Bonhomme, L'Argumentation publicitaire, Paris, Armand Colin, 2014.

2. Le terme produit est pris ici dans le sens extensif que lui donne la publicité, recouvrant tout ce qui fait l'objet d'une promotion médiatique, y compris les réalités géographiques comme les pays touristiques ou les établissements institutionnels comme les banques et les compagnies d'assurances.

3. L'annonceur constitue une instance énonciative générique, englobant non seulement le publicitaire, mais aussi suivant les cas le fabricant et la société qui commercialise le produit. 
4. Traditionnellement, depuis Aristote (Rhétorique, I, 1358b), l'épidictique est envisagé comme l'un des genres de l'éloquence, avec le délibératif et le judiciaire. Néanmoins, il importe d'établir une distinction entre le genre épidictique, historiquement codifié par la rhétorique gréco-latine, et le registre épidictique qui définit le type de discours basé sur l'éloge et qui traverse les époques, ainsi que les cultures. De la sorte, le registre épidictique est exploité dans des genres aussi divers que la harangue politique, l'éditorial journalistique ou la publicité. Pour plus de détails, on peut consulter A. Viala, « Des "registres" ", Pratiques, n 109-110, 2001, p. 165-177; ou M. Bonhomme, "De la pragmatique à la stylistique du registre épidictique », dans L. GaudinBordes et G. Salvan éd., Les Registres, Louvain-la-Neuve, Bruylant-Academia, 2008, p. 79-92.

5. La polarité négative du registre épidictique qu'est le blâme s'avère en effet difficilement compatible avec la rhétorique publicitaire, sauf dans les publicités comparatives et dans les annonces faisant de l'ironie sur elles-mêmes ou feignant de donner la parole à des consommateurs mécontents (voir la section 3.2).

6. M. Dominicy, «L'épidictique et la théorie de la décision », dans M. Dominicy et M. Frédéric éd., La Mise en scène des valeurs, Lausanne/Paris, Delachaux et Niestlé, 2001, p. 49-77.

7. Pour des explications sur ces lieux rhétoriques, définissables comme des stéréotypes logicodiscursifs, on peut se reporter à G. Molinié, Dictionnaire de rhétorique, Paris, Le Livre de Poche, 1992.

8. O. Reboul, La Rhétorique, Paris, PUF, 1984, p. 25.

9. M. Meyer, Questions de rhétorique, Paris, Le Livre de Poche, 1993, p. 35.

10. B. Gibert, La Rhétorique ou les règles de l'éloquence, Paris, D'Espilly, 1749, p. 132.

11. Ch. Perelman et L. Olbrechts-Tyteca, Traité de l'argumentation, Bruxelles, Éditions de l'Université de Bruxelles, 1988.

12. Ch. Plantin, L'Argumentation, Paris, PUF, 2005.

13. J.-B. Grize, «L'argumentation : explication ou séduction », dans L'Argumentation, Lyon, Presses Universitaires de Lyon, 1981, p. 29-40.

14. Le rôle du facteur esthétique dans le domaine épidictique a notamment été relevé par D. Sullivan («The Ethos of Epideictic Encounter », Philosophy and Rhétoric, $\mathrm{n}^{\circ} 26,1993$, p. 113-133).

15. Suivant J.-B. Grize (Logique et langage, Paris, Ophrys, 1990), la schématisation consiste en une représentation sélective et orientée de l'objet du discours en fonction de la situation de communication.

16. Pour R. Barthes («Rhétorique de l'image », Communications, $n^{\circ} 4,1964$, p. 40-51), il existe un rapport de relais entre l'image et le texte lorsque ce dernier retraite, avec la spécificité de son code, une information que l'image a préalablement visualisée.

17. Le lieu de l'« unique » sous-tend un énoncé prônant l'originalité d'une entité ou d'une notion (Ch. Perelman et L. Olbrechts-Tyteca, op. cit.).

18. Ce chiasme consiste à disposer les quatre lettres composant le nom allemand de Berne (Bern) en croisant les couleurs jaune et rouge qui les dessinent.

19. Suivant Aristote (Rhétorique, 1358b, éd. B. Timmermans, Paris, Le Livre de Poche, 1991), « dans une délibération, tantôt l'on conseille, tantôt l'on déconseille ». Mais comme pour le registre épidictique, la publicité ne retient que la facette positive du registre délibératif.

20. À la différence des encarts publicitaires, une plaquette touristique comme celle de Berne est lue par des personnes qui sont déjà intéressées et qui ont fait l'effort de se la procurer. Par conséquent, leur adhésion aura d'autant plus de chance de se réaliser.

21. Ainsi que le note L. Spitzer («La publicité américaine comme art populaire » [1949], Poétique, $\mathrm{n}^{\circ} 34,1978$, p. 152-171), le pôle allocutif vous est ambivalent dans le discours publicitaire, du fait qu'il peut désigner collectivement le public (vous pluriel), mais aussi personnellement chaque lecteur (vous de politesse). Cette ambivalence lui confère une grande résonance médiatique.

22. Respectivement dans Le Système des objets (Paris, Gallimard, 1968, p. 232) et dans La Société de consommation (Paris, Denoël, 1970, p. 263). La «magie du cargo" fait allusion à un mythe 
longtemps répandu chez les indigènes d'Océanie, selon lequel un cargo devait leur apporter une abondance miraculeuse de biens.

23. F. Brune, Le Bonheur conforme, Paris, Gallimard, 1985, p. 148.

24. E. Morin, préface à La Publicité, de l'instrument économique à l'institution sociale de B. Cathelat et A. Cadet, Paris, Payot, 1968, p. 6.

25. J.-P. Teyssier, Frapper sans heurter. Quelle éthique pour la publicité ?, Paris, Armand Colin, 2004, p. 81.

26. Pour cette mouvance appelée aussi greenwashing, voir Th. Libaert, La Communication verte: l'écologie au service de l'entreprise, Paris, Éditions Liaisons, 1992.

27. P. Lemonnier, Quand la publicité est aussi un roman, Paris, Hachette, 1985, p. 176.

28. Apparu à la fin $\mathrm{du} \mathrm{xx}^{\mathrm{e}}$ siècle, ce nouveau courant publicitaire privilégie la dérision, la mise en cause des hiérarchies et le mélange des genres. Pour davantage de précisions, voir N. Riou, Pub Fiction, Paris, Éditions d'organisation, 1999.

29. Sur ce point, se reporter à J.-Cl. Soulages, «Les stratégies humoristiques dans le discours publicitaire ", Questions de communication, n 10, 2006, p. 103-118.

30. Piti est à la fois un jeu de mot paronymique, si on le rapproche de petit, et le verlan de tipi, tente des Indiens d'Amérique du Nord.

31. Voir à ce propos M. Bonhomme, « Parodie et publicité », Tranel, n 44, 2006, p. 165-180.

32. Voici le couplet parodié : « Je ne suis pas notaire, // C'est la faute à Voltaire;// Je suis petit oiseau, // C'est la faute à Rousseau » (Les Misérables, 1862, 1. V).

33. Voir en particulier Cicéron, L'Orateur (éd. A. Yon), Paris, Les Belles Lettres, 1964.

\section{AUTEUR}

\section{MARC BONHOMME}

Université de Berne 